\title{
ON THE GLOBAL DYNAMICS OF A FINANCE MODEL
}

\author{
JAUME LLIBRE ${ }^{1}$ AND CLÀUDIA VALLS ${ }^{2}$
}

\begin{abstract}
Recently several works have studied the following model of finance

$$
\dot{x}=z+(y-a) x, \quad \dot{y}=1-b y-x^{2}, \quad \dot{z}=-x-c z,
$$

where $a, b$ and $c$ are positive real parameters. We study the global dynamics of this polynomial differential system, and in particular for a one-dimensional parametric subfamily we show that there is an equilibrium point which is a global attractor.
\end{abstract}

\section{Introduction AND STATEMENT OF THE RESUlts}

We consider the following polynomial differential system in $\mathbb{R}^{3}$ (see $[1,2$, $3,5,6,7])$

$$
\begin{aligned}
& \dot{x}=z+(y-a) x, \\
& \dot{y}=1-b y-x^{2}, \\
& \dot{z}=-x-c z,
\end{aligned}
$$

where the parameters $a, b, c>0$. This model describes the time variation of three state variables: the interest rate $x$, the investment demand $y$ and the price index $z$. Here $a$ is the saving amount, $b$ is the cost per investment and $c$ is the elasticity of demand of commercial market. The factors that influence changes in $x$ mainly come from an excess of investment over savings and the structural adjustment from good prices. Changing rates in $y$ are in proportion to the rate of investment and in proportion to an inversion with the cost of investment and interest rates. Changes in the variable $z$ are controlled by a contradiction between supply and demand and are influenced by inflation rates.

In this paper we shall provide the complete description of the global dynamics of this polynomial differential system not only on $\mathbb{R}^{3}$ but also in its compactification for some values of the parameters. In this way we will also control the orbits which come or go to infinity. So, this complete information about the dynamics of the polynomial differential system (1) will help to a better understanding of it. More precisely, we wan to describe the

2010 Mathematics Subject Classification. 34C05, 34C07, 34C08.

Key words and phrases. Darboux invariant, finance model, Poincaré compactification, global dynamics. 
$\alpha$-limit sets and the $\omega$-limit sets of all orbits of system (1) for some values of the parameters. Let $\varphi(t)=\varphi(t, p)$ be the solution of system (1) passing through the point $p \in \mathbb{R}^{3}$ when $t=0$, defined on its maximal interval $I_{p}=(\bar{\alpha}(p), \bar{\omega}(p))$. If $\omega(p)=\infty$, then the $\omega$-limit set of $\varphi$ is

$$
\omega(\phi)=\left\{q \in \mathbb{R}^{3}: \exists\left\{t_{n}\right\} \text { with } t_{n} \rightarrow \infty \text { and } \varphi\left(t_{n}\right) \rightarrow q \text { as } n \rightarrow \infty\right\}
$$

Similarly, if $\bar{\alpha}(p)=-\infty$ then the $\alpha$-limit set of $\varphi$ is

$$
\alpha(\phi)=\left\{q \in \mathbb{R}^{3}: \exists\left\{t_{n}\right\} \text { with } t_{n} \rightarrow-\infty \text { and } \varphi\left(t_{n}\right) \rightarrow q \text { as } n \rightarrow \infty\right\}
$$

For more characterizations of the $\alpha$-limit set and of $\omega$-limit set of an orbit, see for instance Section 1.4 of [4].

Note that system (1) is defined in the open manifold $\mathbb{R}^{3}$. For studying its orbits in a neighborhood of the infinity (which has to be done if one wants to study the $\alpha$ and $\omega$-limit sets of the system) we shall identify $\mathbb{R}^{3}$ with the interior of the unit ball

$$
B=\left\{(x, y, z) \in \mathbb{R}^{3}: x^{2}+y^{2}+z^{2} \leq 1\right\}
$$

centered at the origin, and we shall extend analytically this flow to its boundary $\mathbb{S}^{2}$ (the infinity). This compactification is due to Poincaré, and this ball is called the Poincaré ball. The polynomial differential system (1) extended to this closed ball is called the Poincaré compactification of the polynomial differential system (1). For a precise definition of all these notions see the appendix. We shall note that the extended flow to the Poincaré ball leaves invariant the boundary of the ball (the infinity) in the sense that if an orbit has a point in this boundary then the whole orbit is contained in it.

As explained above the infinity is invariant by the Poincaré compactification of system (1). The flow on this boundary (a two dimensional sphere $\mathbb{S}^{2}$ ) is described in the next theorem. For a definition of topologically equivalent phase portraits, see for instance section 1.3 of [4].

Theorem 1. The phase portrait of the Poincaré compactification of system (1) at the infinity $\mathbb{S}^{2}$ is topologically equivalent to the one described in Figure 1 .

Let $\mathbb{R}[x, y, z]$ be the ring of real polynomials in the variables $x, y, z$. We say that $F=F(x, y, z)$ is a Darboux polynomial of system (1) if it satisfies

$$
\frac{\partial F}{\partial x} \dot{x}+\frac{\partial F}{\partial y} \dot{y}+\frac{\partial F}{\partial z} \dot{z}=k F
$$

where $k=k(x, y, z)$ is a real polynomial of degree at most one called the cofactor of $F$ and $(\dot{x}, \dot{y}, \dot{z})$ are given in (1). If the cofactor of $F$ is zero, then $F(x, y, z)$ is a polynomial first integral of system (1). If $F(x, y, z)$ is a Darboux polynomial then the surface $F(x, y, z)=0$ is an invariant algebraic surface. 


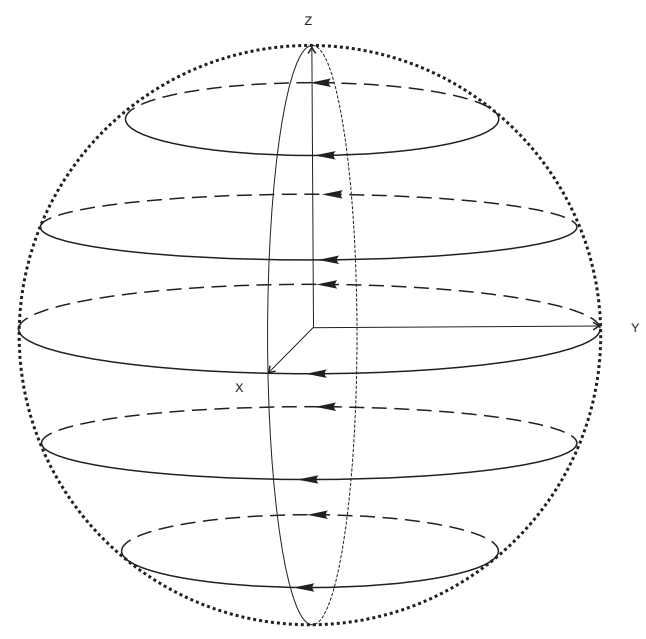

Figure 1. Phase portraits at infinity of system (1). The boundary at infinity of the plane $x=0$.

We say that a $C^{1}$ function $I(x, y, z, t)$ is an invariant of the differential system (1) if $I(x, y, z, t)$ is constant for all values of $t$ for which the solution $(x(t), y(t), z(t)$ is defined. When an invariant function is independent of the time $t$, then it is a first integral.

When a system has a Darboux polynomial $F$ with a constant factor $k=$ $k_{0} \in \mathbb{R}$, then the function $I(x, y, z, t)=F(x, y, z) e^{-k_{0} t}$ is called a Darboux invariant of that system, see Chapter 8 of [4].

Theorem 2. System (1) with

$$
a=-\frac{4+k_{0}^{2}}{2 k_{0}}, \quad b=-\frac{k_{0}}{2}, \quad c=-\frac{k_{0}}{2},
$$

where $k_{0}<0$ has the Darboux polynomial

$$
F_{k_{0}}(x, y, z)=k_{0}^{2} x^{2}+k_{0}^{2} z^{2}+\left(2+k_{0} y\right)^{2}
$$

and the Darboux invariant

$$
I_{k_{0}}(x, y, z, t)=\left(k_{0}^{2} x^{2}+k_{0}^{2} z^{2}+\left(2+k_{0} y\right)^{2}\right) e^{-k_{0} t} .
$$

For the values of the parameters given in (2) we have that $F_{k_{0}}=0$ is an invariant algebraic surface. 
For the values $(a, b, c)$ given in (2), system (1) can be written as

$$
\begin{aligned}
& \dot{x}=\frac{4+k_{0}^{2}}{2 k_{0}} x+z+x y, \\
& \dot{y}=1+\frac{k_{0} y}{2}-x^{2}, \\
& \dot{z}=-x+\frac{k_{0} z}{2},
\end{aligned}
$$

where $k_{0}<0$. In the following theorem we describe the dynamics of system (3). We recall that a point $p$ is globally asymptotically stable for system (3) if every solution $(x(t), y(t), z(t))$ of system (3) is defined for $t \rightarrow \infty$ and tends to $p$ when $t \rightarrow \infty$.

Theorem 3. For the values of the parameters $(a, b, c)$ given in (2) the phase portrait of system (3) in $\mathbb{R}^{3}$ is as follows: the invariant algebraic surface $F_{k_{0}}(x, y, z)=0$ is formed by the point $q=\left(0,-2 / k_{0}, 0\right)$, which is the unique equilibrium point of system (3), and it is globally asymptotically stable.

In the next theorem we describe the $\alpha$ - and the $\omega$-limit sets of all orbits contained in $B$ of system (3). Let $P$ be a diffeomorphism such that $P\left(\mathbb{R}^{3}\right)$ is equal to the interior of the Poincaré ball. Then we denote the finite isolated singular point $q$ of system (3) given in Theorem 3 as $p=P(q)$.

Theorem 4. Let $\gamma$ be an orbit of system (3).

(a) If $\gamma$ is contained in the boundary of the Poincaré ball $B$ and is different from a singular point, then its $\alpha$-and $\omega$-limit sets are different infinite singular points.

(b) If $\gamma$ is contained in the interior of $B$ and is different from the singular point $q=\left(0,-2 / k_{0}, 0\right)$, then the following two statement hold.

(b.1) The $\alpha$-limit set of $\gamma$ is an infinite singular point.

(b.2) The $\omega$-limit set of $\gamma$ is the unique finite singular point $q$.

Note that in both cases such a $\gamma$ is a heteroclinic orbit.

The proofs of all theorems are given in the next section.

We remark that Theorem 1 works for system (1), i.e. for the system with the three parameters $a, b$ and $c$. While the other theorems only work for the one-dimensional subfamily of systems (1) defined by condition (2).

\section{Proof of the Results}

Proof of Theorem 1. For studying the infinity of the Poincaré ball $B$ we analyze the flow at infinity for the local charts $U_{1}, U_{2}$ and $U_{3}$, see the appendix. 
In the local chart $U_{1}$ system (1) writes

$$
\begin{aligned}
& \dot{z}_{1}=-1-z_{1}^{2}+(a-b) z_{1} z_{3}+z_{3}^{2}-z_{1} z_{2} z_{3}, \\
& \dot{z}_{2}=-z_{3}-z_{1} z_{2}+(a-c) z_{2} z_{3}-z_{2}^{2} z_{3}, \\
& \dot{z}_{3}=z_{3}\left(-z_{1}+a z_{3}-z_{2} z_{3}\right) .
\end{aligned}
$$

System (4) restricted at the infinity (that is with $z_{3}=0$ ) becomes

$$
\dot{z}_{1}=-1-z_{1}^{2}, \quad \dot{z}_{2}=-z_{1} z_{2} .
$$

so there are no singular points at infinity in the local chart $U_{1}$.

In the local chart $U_{2}$ system (1) writes

$$
\begin{aligned}
& \dot{z}_{1}=z_{1}-(a-b) z_{1} z_{3}+z_{2} z_{3}+z_{1}^{3}-z_{1} z_{3}^{2}, \\
& \dot{z}_{2}=-z_{1} z_{3}+(b-c) z_{2} z_{3}+z_{1}^{2} z_{2}-z_{2} z_{3}^{2}, \\
& \dot{z}_{3}=z_{3}\left(b z_{3}+z_{1}^{2}-z_{3}^{2}\right) .
\end{aligned}
$$

System (5) restricted at the infinity (that is with $z_{3}=0$ ) becomes

$$
\dot{z}_{1}=z_{1}\left(1+z_{1}^{2}\right), \quad \dot{z}_{2}=z_{1}^{2} z_{2},
$$

so there is a line of singular points which is $z_{1}=0$. After eliminating the common factor $z_{1}$ (by a rescaling of the time) we do not obtain any singular point in the local chart $U_{2}$ which is distinguished.

In the local chart $U_{3}$ system (1) writes

$$
\begin{aligned}
& \dot{z}_{1}=z_{3}+z_{1} z_{2}+(c-a) z_{1} z_{3}+z_{1}^{2} z_{3}, \\
& \dot{z}_{2}=-z_{1}^{2}+(c-b) z_{2} z_{3}+z_{3}^{2}+z_{1} z_{2} z_{3}, \\
& \dot{z}_{3}=z_{3}^{2}\left(c+z_{1}\right) .
\end{aligned}
$$

Now the unique point of the local chart $U_{3}$ which is not covered by the local charts $U_{1}, V_{1}, U_{2}$ and $V_{2}$ is the origin of coordinates of $U_{3}$. System (6) restricted at the infinity (that is with $z_{3}=0$ ) becomes

$$
\dot{z}_{1}=z_{1} z_{2}, \quad \dot{z}_{2}=-z_{1}^{2} \text {. }
$$

Since the origin of the previous system is a singular point, we have that the circle at infinity in the boundary of the plane $x=0$ is filled of singular points. After eliminating the common factor $z_{1}$ (by a rescaling of the time) we get that the origin is a center.

After gluing all the information we have on the local charts $U_{1}, U_{2}$ and $U_{3}$, we get that the phase portrait of system (3) at infinity of $B$ is defined in Figure ??.

Proof. Proof of Theorem 2 Since $F_{k_{0}}(x, y, z)=k_{0}^{2} x^{2}+k_{0}^{2} z^{2}+\left(2+k_{0} y\right)^{2}$ it is immediate that when $(a, b, c)$ are the ones given in (2) then

$$
\frac{d F_{k_{0}}}{d t}=\frac{\partial F_{k_{0}}}{\partial x} \dot{x}+\frac{\partial F_{k_{0}}}{\partial y} \dot{y}+\frac{\partial F_{k_{0}}}{\partial z} \dot{z}=k_{0} F_{k_{0}},
$$


where $(\dot{x}, \dot{y}, \dot{z})$ are given in (3). Therefore $F_{k_{0}}$ is a Darboux polynomial with cofactor $k_{0}$.

Moreover $I_{k_{0}}(x, y, z, t)=F_{k_{0}}(x, y, z, t) e^{-k_{0} t}$ satisfies

$$
\frac{d I_{k_{0}}}{d t}=\frac{\partial I_{k_{0}}}{\partial t}+\frac{\partial I_{k_{0}}}{\partial x} \dot{x}+\frac{\partial I_{k_{0}}}{\partial y} \dot{y}+\frac{\partial I_{k_{0}}}{\partial z} \dot{z}=-k_{0} F_{k_{0}}+\frac{d F_{k_{0}}}{d t}=0,
$$

where again $(\dot{x}, \dot{y}, \dot{z})$ are given in (3). This completes the proof of Theorem 2 .

Proof of Theorem 3. The invariant algebraic surface $k_{0}^{2} x^{2}+k_{0}^{2} z^{2}+\left(k_{0} y+\right.$ $2)^{2}=0$ reduces to the point $q=\left(0,-2 / k_{0}, 0\right)$ on the $y$-axis. The point $q=\left(0,-2 / k_{0}, 0\right)$ is the unique equilibrium point of the system and is a stable equilibrium point (locally attractor) because the eigenvalues of the linear part at this singular point are $k_{0} / 2,\left(k_{0} \pm 2 i\right) / 2$. Moreover, we claim that the unique equilibrium point $q$ of system (3) is globally asymptotically stable because it has the global Lyapunov function

$$
H(x, y, z)=x^{2}+z^{2}+\frac{4}{k_{0}^{2}}\left(1+\frac{k_{0} y}{2}\right)^{2} .
$$

Indeed, from Theorem 1.35 of [4] we only need to show that the derivative of $H$ with respect to the independent variable $t$, over the solutions of system (3) with $(a, b, c)$ as in the statement of Theorem 2 is negative, and this is the case because

$$
\frac{d H}{d t}=k_{0} x^{2}+k_{0} z^{2}+\frac{4}{k_{0}}\left(1+\frac{k_{0} y}{2}\right)^{2}
$$

and $k_{0}<0$.

Proof of Theorem 4. Clearly statement (a) of Theorem 4 follows from Theorem 1. Statement (b) follows from Theorem 3.

\section{Appendix: Poincaré COMPACtifichtion In $\mathbb{R}^{3}$}

In $\mathbb{R}^{3}$ we consider the polynomial differential system

$$
\dot{x}=P_{1}(x, y, z), \quad \dot{y}=P_{2}(x, y, z), \quad \dot{z}=P_{3}(x, y, z)
$$

or equivalently its associated polynomial vector field $X=\left(P_{1}, P_{1}, P_{3}\right)$. The degree $n$ of $X$ is defined as $n=\max \left\{\operatorname{deg}\left(P_{i}\right): i=1,2,3\right\}$. Let

$$
\mathbb{S}^{3}=\left\{y=\left(y_{1}, y_{2}, y_{3}, y_{4}\right) \in \mathbb{R}^{4}:\left\|y_{4}\right\|=1\right\}
$$

be the unit sphere in $\mathbb{R}^{4}$ and

$$
H_{+}=\left\{y \in \mathbb{S}^{3}: y_{4}>0\right\}, \quad H_{-}=\left\{y \in \mathbb{S}^{3}: y_{4}<0\right\}
$$

be the northern and southern hemispheres, respectively. The tangent space to $\mathbb{S}^{3}$ at the point $y$ is denoted by $T_{y} \mathbb{S}^{3}$. The tangent hyperplane

$$
T_{(0,0,0,1)} \mathbb{S}^{3}=\left\{\left(x_{1}, x_{2}, x_{3}, 1\right) \in \mathbb{R}^{4}:\left(x_{1}, x_{2}, x_{3}\right) \in \mathbb{R}^{3}\right\}
$$


is identified with $\mathbb{R}^{3}$. We consider the projections

$$
f_{+}: \mathbb{R}^{3} \rightarrow H_{+}, \quad f_{-}: \mathbb{R}^{3} \rightarrow H_{-},
$$

defined by

$$
\begin{aligned}
& f_{+}(x)=\frac{1}{\left(1+\sum_{i=1}^{3} x_{i}^{2}\right)^{1 / 2}}\left(x_{1}, x_{2}, x_{3}, 1\right), \\
& f_{-}(x)=-\frac{1}{\left(1+\sum_{i=1}^{3} x_{i}^{2}\right)^{1 / 2}}\left(x_{1}, x_{2}, x_{3}, 1\right) .
\end{aligned}
$$

Through these projections $\mathbb{R}^{3}$ can be identified with the northern and the southern hemispheres, respectively. The equator of $\mathbb{S}^{3}$ is $\mathbb{S}^{2}=\left\{y \in \mathbb{R}^{4}\right.$ : $\left.y_{4}=0\right\}$ is identified with the infinity of $\mathbb{R}^{3}$.

The maps $f_{+}$and $f_{-}$define two copies of $X$, one $D f_{+} \circ X$ in the northern hemisphere and the other $D f_{-} \circ X$ in the southern hemisphere. We denote by $\bar{X}$ the vector field in $\mathbb{S}^{3} \backslash \mathbb{S}^{2}=H_{+} \cup H_{-}$that restricted to $H_{+}$coincides with $D f_{+} \circ X$ and restricted to $H_{-}$coincides with $D f_{-} \circ X$.

We will work with the orthogonal projection of the closed northern hemisphere to $y_{4}=0$. Note that this projection is the closed Poincaré ball $B$, whose interior is homeomorphic to $\mathbb{R}^{3}$ and whose boundary $\mathbb{S}^{2}$ corresponds to the infinity of $\mathbb{R}^{3}$. We will extend analytically the polynomial vector field $\bar{X}$ to the boundary, in such a way that the flow on the boundary will become invariant. This new vector field on $B$ is called the Poincaré compactification $P(X)$ of $X$.

The expression for $\bar{X}(y)$ on $H_{+} \cup H_{-}$is

$$
\bar{X}(y)=y_{4}\left(\begin{array}{ccc}
1-y_{1}^{2} & -y_{2} y_{1} & -y_{3} y_{1} \\
-y_{1} y_{2} & 1-y_{2}^{2} & -y_{3} y_{2} \\
-y_{1} y_{3} & -y_{2} y_{3} & 1-y_{3}^{2} \\
-y_{1} y_{4} & -y_{2} y_{4} & -y_{3} y_{4}
\end{array}\right)\left(\begin{array}{l}
P_{1} \\
P_{2} \\
P_{3}
\end{array}\right),
$$

where $P_{i}=P_{i}\left(y_{1} /\left|y_{4}\right|, y_{2} /\left|y_{3}\right|, y_{1} /\left|y_{4}\right|\right)$ for $i=1,2,3$. In this way $\bar{X}(y)$ is a vector field that is tangent to the sphere $\mathbb{S}^{3}$. Now we extend analytically the vector field $\bar{X}(y)$ to a vector field $p(X)$ defined in the whole sphere $\mathbb{S}^{3}$ doing

$$
p(X)(y)=y_{4}^{n-1} \bar{X}(y) .
$$

This extended vector field is called the Poincaré compactification of $X$.

Since $\mathbb{S}^{3}$ is a differentiable manifold, to compute the expression of $p(X)$ we can consider the eight local charts $\left(U_{i}, F_{i}\right),\left(V_{i}, G_{i}\right)$ where

$$
U_{i}=\left\{y \in \mathbb{S}^{3}: y_{i}>0\right\}, \quad V_{i}=\left\{y \in \mathbb{S}^{3}: y_{i}<0\right\},
$$

for $i=1,2,3,4$. The diffeomorphisms $F_{i}: U_{i} \rightarrow \mathbb{R}^{3}$ and $G_{i}: V_{i} \rightarrow \mathbb{R}^{3}$ are the inverses of the projections from the origin to the tangent planes at the points $( \pm 1,0,0,0),(0, \pm 1,0,0),(0,0, \pm 1,0)$ and $(0,0,0, \pm 1)$, respectively. 
The analytical expression of the vector field $p(X)$ in the local charts $U_{1}$, $U_{2}$ and $U_{3}$ are (after a rescaling of time that eliminates the factor $((1+$ $\left.\left.\left.\sum_{i=1}^{3} z_{i}^{3}\right)^{1 / 2}\right)^{n-1}\right)$

$$
z_{3}^{n}\left(-z_{1} P_{1}+P_{2},-z_{2} P_{1}+P_{3},-z_{3} P_{1}\right)
$$

where $P_{i}=P_{i}\left(1 / z_{3}, z_{1} / z_{3}, z_{2} / z_{3}\right)$ in $U_{1}$,

$$
z_{3}^{n}\left(-z_{1} P_{2}+P_{1},-z_{2} P_{2}+P_{3},-z_{3} P_{2}\right)
$$

where $P_{i}=P_{i}\left(z_{1} / z_{3}, 1 / z_{3}, z_{2} / z_{3}\right)$ in $U_{2}$, and

$$
z_{3}^{n}\left(-z_{1} P_{3}+P_{1},-z_{2} P_{3}+P_{2},-z_{3} P_{3}\right)
$$

where $P_{i}=P_{i}\left(z_{1} / z_{3}, z_{2} / z_{3}, 1 / z_{3}\right)$ in $U_{3}$, respectively. The expression for $p(X)$ in $U_{4}$ is $z_{3}^{n+1}\left(P_{1}, P_{2}, P_{3}\right)$ where $P_{i}=P_{i}\left(z_{1}, z_{2}, z_{3}\right)$. The expression of $p(X)$ on the local charts $V_{i}$ is the same as in the local charts $U_{i}$ multiplied by $(-1)^{n-1}$. Note that all the points on the sphere at infinity in the coordinates of any local chart have $z_{3}=0$.

\section{ACKNOWLEDGEMENTS}

The first author is partially supported by a FEDER-MINECO grant MTM2016-77278-P, a MINECO grant MTM2013-40998-P, and an AGAUR grant number 2014SGR-568. The second author is partially supported by FCT/Portugal through UID/MAT/04459/2013.

\section{REFERENCES}

[1] A. Arikoglu and I. Ozkol, Solution of fractional integro-differential equations by using fractional differential transform method, Chaos, Solitons \& Fractals 40 (2009), 521-529.

[2] M.K. Bouafoura, O. Moussi, O And N.B. Braiek, A fractional state space realization method with block pulse basis, Signal. Process. 91 (2011), 492-497.

[3] A. Deb, A. Dasgupta And G. Sarkar, A new set of orthogonal functions and its application to the analysis of dynamic systems, J. Frankl. Inst. 343 (2006), 1-26.

[4] F. Dumortier, J. Llibre And J.C. Artés, Qualitative Theory of Planar Differential Systems, Springer Verlag, New York, 2006.

[5] H.R. Fazli, F. Hassani, A. Ebadian And A.A. Khajehnasiri, National economies in state-space of fractional-order financial system, Afr. Mat. 27 (2016), 529-540.

[6] S. Momani And M.A. Noor, Numerical methods for fourth-order fractional integrodifferential equations, Appl. Math. Comput. 182 (2006), 754-760.

[7] T. Skovrnek, I., Podlubny And I. Petrs, Modeling of the national economies in state-space: a fractional calculus approach, Econ. Model. 29 (2012), 1322-1327.

\footnotetext{
${ }^{1}$ Departament de Matemàtiques, Universitat Autònoma de Barcelona, 08193 Bellaterra, Barcelona, Catalonia, Spain
}

E-mail address: jllibre@mat.uab.cat 
2 Departamento de Matemática, Instituto Superior Técnico, Universidade de Lisboa, Av. Rovisco Pais 1049-001, Lisboa, Portugal

E-mail address: cvalls@math.ist.utl.pt 